\title{
Comparative review of outcomes of transabdominal preperitoneal (TAPP) and totally extraperitoneal (TEP) Rives-Stoppa in robotic ventral hernia repair
}

\author{
Omar Yusef Kudsi, Naseem Bou-Ayash, Fahri Gokcal \\ Department of Surgery, Good Samaritan Medical Center, Tufts University Faculty of Medicine, Brockton, Boston, MA, USA
}

\begin{abstract}
Introduction: Extraperitoneal approaches to ventral hernia repair (VHR) utilize the inner layers of the abdominal wall as a barrier. The robotic approach is promising in that it provides repair quality similar to its open and laparoscopic counterparts, with a decreased perioperative morbidity. Our aim is to compare the short-term outcomes between robotic totally extraperitoneal Rives-Stoppa (rTEP-RS) and transabdominal preperitoneal (rTAPP) VHR.

Materials and Methods: A comparative analysis was performed in terms of perioperative and early outcomes. Univariate tests were used to compare two groups. A subset analysis of all variables was conducted in patients with and without complications. A logistic regression analysis was used to determine factors affecting the presence of postoperative complications.

Results: From 598 patients, 63 patients underwent rTEP-RS and 143 patients underwent rTAPP VHR. There were no differences between the groups in terms of patient demographics. The average defect size, mesh size and overlap were higher in the rTEP-RS group. Operative times were longer in the rTEP group. There were no differences between the two groups in terms of post-operative outcomes including complication rates and surgical site events. Female sex and console time were associated with postoperative complications.

Conclusion: This is the largest study to date comparing the rTEP-RS and rTAPP approaches to VHR. The short-term results for rTEP-RS repair were similar rTAPP repair. The rTEP-RS approach allowed for large hernias defects to be repaired with large-sized mesh.
\end{abstract}

Keywords: Retrorectus; retromuscular; robotics; transabdominal preperitoneal; TAPP; totally extraperitoneal; TEP; rivesstoppa; ventral hernia repair.

\section{Introduction}

The efficacy of ventral hernia repair (VHR) has been improving alongside the technological advancements in this field. The introduction of minimally invasive techniques has allowed for the exploration of different mesh positions, suturing and fixation methods, and surgical access. Laparoscopic intraperitoneal onlay mesh (IPOM) placement was first described by LeBlanc and Booth, ${ }^{[1]}$ and 
several studies have reported decreased wound complications, faster recovery, and lower recurrence rates with this technique as compared to open repair.

As the adoption of the IPOM technique increased, so did the observation of its related complications such as adhesion formation and mesh erosion. ${ }^{[2,3]}$ This eventually led surgeons to explore other extraperitoneal mesh positions. By using the peritoneum as a barrier between the mesh and the abdominal cavity, surgeons are able to avoid the abovementioned complications as well as the need for mesh fixation.

The robotic platform facilitates both preperitoneal and retromuscular mesh placements, which have promising results in terms of complication rates and hospital length of stay. ${ }^{[4,5]}$ Although previous studies have favored robotic transabdominal preperitoneal (rTAPP) and totally extraperitoneal Rives-Stoppa (rTEP-RS) techniques over robotic IPOM repair in terms of early postoperative outcomes, ${ }^{[6,7]}$ these two techniques have not been compared directly. In this study, we aim to compare the short-term outcomes of rTAPP and rTEP-RS techniques.

\section{Materials and Methods}

The data of this study was obtained from both a prospectively maintained database and electronic medical records of patients who underwent robotic ventral hernia procedures at a single center between February 2013 and December 2019. The database used for this study approved by the Institutional Review Board and informed consent was obtained from all individual participants included in the study. The quality and completeness of the database was externally validated by a researcher. Data was reviewed in terms of preoperative, intraoperative, and postoperative variables. Preoperative variables included patient demographics, hernia etiology, hernia location, the American Society Anesthesiologists classification scores (ASA), comorbidities, risk factors, and procedure setting. Operative details included surgical technique, adhesiolysis time greater than 30 minutes, primary closure of the hernia defect, type of mesh materials, mesh fixation methods, hernia defect dimensions, mesh dimensions, operative time (console, skinto-skin), estimated blood loss (EBL), and intraoperative complications. European Hernia Society (EHS) ${ }^{[8]}$ recommendations were followed to categorize hernia location and to measure the defect size. The defect area $\left(\mathrm{cm}^{2}\right)$, mesh area $\left(\mathrm{cm}^{2}\right)$, mesh overlap, and ratio of mesh to de- fect size (M/D ratio) were determined using conventional mathematical formulas, which have been previously described. ${ }^{[7]}$ Postoperative variables were defined as follows: postoperative pain scores (0-to-10 verbal scale assessed immediately after surgery in post-anesthesia care unit-PACU), hospital length of stay (LOS), and hospital readmission within 30-days. As part of routine patient care, all patients were clinically evaluated at mainly two intervals post-operatively; the first was performed within three weeks and the second within three months. For this study, a follow-up of up to 90-days was chosen to ensure detection of postoperative surgical complications related to index procedures.

All postoperative complications were categorized according to the Clavien-Dindo classification system. ${ }^{[9]}$ The morbidity score was measured using the Comprehensive Complication Index (CCI®, University of Zurich, Zurich, Switzerland). ${ }^{[10]}$ Surgical wound complications were categorized according to the previously published classifications. ${ }^{[11]}$ Briefly, surgical site events (SSEs) were defined as surgical site infections (SSIs), surgical site occurrences (SSOs), and surgical site occurrences requiring procedural intervention (SSOPI). SSIs were further classified as cellulitis, superficial, deep, and organ space infections. SSOs included sterile fluid collections such as hematomas and seromas. Any SSO or SSI requiring procedural intervention such as percutaneous puncturing to reduce symptoms, bedside wound opening, or reoperation, was described as an SSOPI.

Follow-up of complications up to ninety days was performed by reviewing prospectively maintained records, medical records for both in- and outpatient clinic visits, as well as emergency department visits.

From the cohort of robotic ventral hernia repairs, only patients who underwent rTEP-RS and rTAPP VHR were included in the study. Patients who underwent concomitant procedures, adjunctive transversus abdominis release (TAR), robotic intraperitoneal onlay (rIPOM) mesh repair, and transabdominal retromuscular (rTA-RM) mesh repair were excluded.

\section{Surgical Technique}

For rTEP-RS repair, initial trocar placement was performed laparoscopically using optical trocar entry. After the other trocars were placed under direct vision, the patient-side cart of the robot was docked and the remainder of the surgery was achieved robotically. Upon completion 
of the ipsilateral retrorectus dissection, the medial edge of the rectus sheath was incised in order to reach the contralateral rectus sheath. Once the preperitoneal dissection at the posterior aspect of the linea alba was achieved, the medial border of the contralateral rectus sheath was incised to merge the retrorectus spaces together into one compartment (crossover) that is enclosed by the linea semilunaris on both sides. Primary closure of the anterior fascial defect was then performed by running a long-lasting absorbable barbed suture and the mesh was deployed in the retrorectus space. Any defects in the posterior flap were then closed using absorbable suture. Skin incisions were closed with absorbable sutures.

For rTAPP repair, two working ports and a single camera port were used. Based on the hernia defect size, the appropriate mesh size was chosen to obtain the necessary overlap. Using monopolar scissors and a bipolar grasper, the peritoneum on the side ipsilateral to the trocars was grasped and cut to enter the preperitoneal space at least 5 $\mathrm{cm}$ away from the defect. Preperitoneal dissection was extended at least $5 \mathrm{~cm}$ circumferentially around the defect in order to provide adequate mesh overlap. The intraabdominal pressure was then reduced $(6-8 \mathrm{mmHg})$ and primary closure of the hernia defect was achieved by running a long-lasting absorbable barbed suture (Stratafix $0^{\mathrm{TM}}$ on CT-1 needle, Ethicon, Somerville, NJ, USA), taking 5-8 mm bites of fascia every $5 \mathrm{~mm}$ in a running fashion. The mesh was secured to the posterior fascia with an absorbable suture. In case of failure to maintain an intact peritoneal flap, coated meshes were used. Otherwise, uncoated meshes were used. If present, small tears in the peritoneum were repaired using absorbable sutures. The peritoneal flap was closed with a barbed absorbable suture (2-0 V-Loc ${ }^{\mathrm{TM}}$; Medtronic, Minneapolis, MN, USA). Dehiscence was assessed by increasing intraabdominal pressure to $15 \mathrm{~mm} \mathrm{Hg}$. The patient-side cart was then undocked. The trocars were removed with release of pneumoperitoneum. The fascia for trocar sites $10 \mathrm{~mm}$ or larger was sutured to decrease the risk of future incisional hernias. Long acting local anesthetic was injected in the incisions for postoperative pain management. ${ }^{[7]}$

\section{Statistical Analysis}

Categorical variables were presented as the frequency with percentage $[\mathrm{n}(\%)]$ and continuous variables as mean $\pm \mathrm{SD}$ or median (interquartile range, IQR), as appropriate. Categorical variables were analyzed using Pearson Chi-Square or Fisher`s Exact Tests. Continuous variables were ana- lyzed using the Independent-Sample t-test (for normal distributions) or Mann-Whitney U Test (for non-normal distributions). A subset analysis of all variables was examined in patients with and without complications. A logistic regression analysis was conducted to determine which factors affect the presence of postoperative complications. Statistical assessments were performed using SPSS software pack (Statistical Package for Social Sciences for Windows version 22 software). A p-value of $<0.05$ was considered statistically significant.

\section{Results}

Out of the initial cohort of 598 consecutive patients who underwent rVHR, 206 patients who underwent rTAPP and rTEP-RS repair were included in the study. Of these, rTAPP repair was performed in 143 (69.4\%) patients and rTEP-RS repair was performed in $63(30.6 \%)$ patients. The patient selection flowchart is displayed in Figure 1.

The comparison of preoperative variables is shown in Table 1. Accordingly, the average age of patients was higher in the rTEP-RS group. All hernias were located at the midline except for $8(5.6 \%)$ patients who had an off-midline hernia in the rTAPP group $(\mathrm{p}=0.056 ; 0 \%$ in rTEP-RS).

In terms of hernia content, omentum was noted in $84.1 \%$ of the rTEP-RS patients versus $58 \%$ of the rTAPP patients $(\mathrm{p}=<0.001)$. Colon was found in $5(3.5 \%)$ of rTAPP patients ( $\mathrm{p}=0.133 ; 0 \%$ of the rTEP-RS group). Small bowel was noted among 7 (4.9\%) rTAPP patients versus $2(3.2 \%)$ rTEP-RS patients ( $\mathrm{p}=0.578$ ). Significantly larger defect size and mesh size, as well as less mesh fixation were noted in the rTEP-RS group. Both console and skin-to-skin times were shorter in the rTAPP group. While a gastric serosal injury occurred in $1(0.7 \%)$ patient who underwent rTAPP

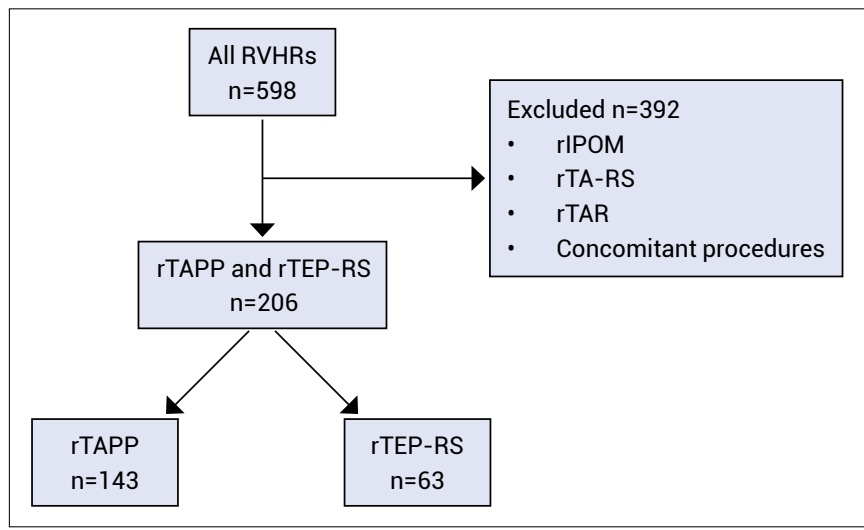

Figure 1. Patient selection flowchart. 
Table 1. Comparison of preoperative variables between the two groups

\begin{tabular}{|c|c|c|c|}
\hline & $\begin{array}{c}\text { rTAPP } \\
(n=143)\end{array}$ & $\begin{array}{c}\text { rTEP-RS } \\
(n=63)\end{array}$ & $\mathbf{p}$ \\
\hline Age (years), mean $\pm S D$ & $51.3 \pm 13.8$ & $55.7 \pm 14.5$ & 0.042 \\
\hline Sex, female, $\mathrm{n}(\%)$ & $95(66.4)$ & $45(71.4)$ & 0.520 \\
\hline BMI $\left(\mathrm{kg} / \mathrm{m}^{2}\right)$, mean $\pm S D$ & $31.8 \pm 6.3$ & $30.3 \pm 5.6$ & 0.133 \\
\hline ASA Score, median (IQR) & $2(2-3)$ & $2(2-3)$ & 0.074 \\
\hline \multicolumn{4}{|l|}{ Risk factors, $\mathrm{n}(\%)$} \\
\hline HT, yes & $67(46.9)$ & $31(49.2)$ & 0.764 \\
\hline MI, yes & $1(0.7)$ & $1(1.6)$ & 0.549 \\
\hline CAD, yes & $7(4.9)$ & $6(9.5)$ & 0.208 \\
\hline DM, yes & $20(14)$ & $13(20.6)$ & 0.302 \\
\hline COPD, yes & $15(10.5)$ & $4(6.3)$ & 0.439 \\
\hline Smoking, yes & $36(25.2)$ & $9(14.3)$ & 0.100 \\
\hline History of wound infection, yes & $3(2.1)$ & $3(4.8)$ & 0.295 \\
\hline Immunosuppression, yes & $2(1.4)$ & $2(3.2)$ & 0.395 \\
\hline MVHWG grades, median (IQR) & $2(2-2)$ & $2(2-2)$ & 0.698 \\
\hline HPW stages, median (IQR) & $2(2-2)$ & $2(2-2)$ & 0.630 \\
\hline \multicolumn{4}{|l|}{ Hernia etiology, n (\%) } \\
\hline Primary ventral & $110(75.9)$ & $49(77.8)$ & \\
\hline Incisional & $33(23.1)$ & $14(22.2)$ & 1.000 \\
\hline Recurrent hernia, yes, n (\%) & $14(9.8)$ & $7(11.1)$ & 0.805 \\
\hline Procedure setting, elective, $\mathrm{n}(\%)$ & $137(95.8)$ & $59(93.7)$ & 0.508 \\
\hline
\end{tabular}

repair during trocar insertion, there were no intraoperative complications in the rTEP-RS group $(\mathrm{p}=0.506)$. A drain was not placed in any of the procedures. No patients required conversion to an open or laparoscopic approach. Intraoperative findings are summarized in Table 2.

In terms of postoperative pain prior to leaving the PACU, the median (IQR) postoperative pain scores did not differ significantly between the two groups $[\mathrm{p}=0.068 ; 3(3-5)$ rTAPP vs. 4 (3-6) rTEP-RS]. However, the median (IQR) milligram morphine equivalent was significantly higher in the rTAPP group than the rTEP-RS group [p $<0.001 ; 10.7$ (10-20) vs. 5 (0-10.5), respectively]. The median (range) LOS did not differ between groups $[\mathrm{p}=0.281 ; 0(0-4)$ days for rTAPP and 0 (0-6) days for rTEP-RS].

The average follow-up time was 33 (range=2.7-61.9) months for the entire cohort. In terms of 30-day hospital readmission, there was no difference between the two groups ( $\mathrm{p}=0.917)$; while $2(1.4 \%)$ patients in the rTAPP group were readmitted due to small bowel obstruction, 1 $(1.6 \%)$ patient in the rTEP-RS group was readmitted due to a hematoma. All patients were managed conservatively. No hernia recurrence was seen in both groups during the 90-day follow-up period. 14 (11\%) patients in the rTAPP group and 4 (7.4\%) patients in rTEP-RS group did not attend their postoperative visits. Accordingly, postoperative outcomes were assessed in a total of 181 patients. The overall proportion of patients with any postoperative complication did not differ between groups ( $\mathrm{p}=0.592 ; 11 \%$ vs. 7.4\%, respectively). Types and severity of postoperative complications are summarized in Table 3. In the rTAPP group, one patient required percutaneous drainage due to a surgical site hematoma. The rate of SSEs, SSOs and SSIs did not differ between groups.

The development of complications was associated with female gender and console time according to a regression analysis corrected for age and hernia content. The results are shown in Table 4. 
Table 2. Comparison of intraoperative variables between the two groups

\begin{tabular}{|c|c|c|c|c|c|}
\hline & \multicolumn{2}{|c|}{$\begin{array}{c}\text { rTAPP } \\
(n=143)\end{array}$} & \multicolumn{2}{|c|}{$\begin{array}{c}\text { rTEP-RS } \\
(n=63)\end{array}$} & \multirow[t]{2}{*}{$\mathbf{p}$} \\
\hline & $\mathbf{n}$ & $\%$ & $\mathbf{n}$ & $\%$ & \\
\hline Adhesiolysis (>30 min) & 6 & 4.2 & 0 & 0 & 0.099 \\
\hline Defect size, $\mathrm{cm}^{2}$, median (IQR) & 3.1 & $3.1-7$ & 15.7 & $11.8-18.8$ & $<0.001$ \\
\hline Primary defect closure, yes & 133 & 93 & 63 & 100 & 0.031 \\
\hline Mesh size, $\mathrm{cm}^{2}$, median (IQR) & 113 & $63.6-180$ & 225 & $225-300$ & $<0.001$ \\
\hline Mesh overlap, cm, median (IQR) & 4.5 & $3.5-5$ & 5.5 & $5.5-6$ & $<0.001$ \\
\hline Mesh/Defect ratio, median (IQR) & 19.2 & $12-35$ & 11.5 & $10.2-17.7$ & $<0.001$ \\
\hline \multicolumn{6}{|l|}{ Mesh materials } \\
\hline Polypropylene & 13 & 9.1 & 47 & 74.6 & \\
\hline Polyester & 127 & 88.8 & 3 & 4.8 & \\
\hline ePTFE, & 3 & 2.1 & 13 & 20.6 & $<0.001$ \\
\hline Mesh fixation, yes & 109 & 76.2 & 3 & 4.8 & $<0.001$ \\
\hline Console time, minutes, median (IQR) & 42 & $32-52$ & 57 & $41-80.5$ & $<0.001$ \\
\hline Skin-to-skin time, minutes, median (IQR) & 56 & $44-69.5$ & 72 & $53.5-99.5$ & $<0.001$ \\
\hline $\mathrm{EBL}, \mathrm{mL}$, median (IQR) & 5 & 5-5 & 5 & $5-5$ & 0.621 \\
\hline
\end{tabular}

rTAPP. robotic transabdominal preperitoneal; rTEP-RS: robotic total extraperitoneal Rives-Stoppa; ePTFE: expanded polytetrafluoroethylene; EBL: estimated blood loss; IQR: interquartile range.

\section{Table 3. Comparison of postoperative variables between the two groups}

\begin{tabular}{|c|c|c|c|c|c|}
\hline & \multicolumn{2}{|c|}{$\begin{array}{l}\text { rTAPP } \\
(n=127)\end{array}$} & \multicolumn{2}{|c|}{$\begin{array}{c}\text { rTEP-RS } \\
(n=54)\end{array}$} & \multirow[t]{2}{*}{$\mathbf{p}$} \\
\hline & $\mathbf{n}$ & $\%$ & $\mathbf{n}$ & $\%$ & \\
\hline $\mathrm{CCl} \AA^{\circledR}$, median (range) & 0 & $0-34.8$ & 0 & 20.9 & 0.413 \\
\hline \multicolumn{6}{|l|}{ Clavien-Dindo } \\
\hline Grade-1 & 7 & 5.5 & 3 & 5.6 & \\
\hline Grade-2 & 4 & 3.1 & 1 & 1.9 & \\
\hline Grade-3a & 2 & 1.6 & 0 & 0 & \\
\hline Grade-3b & 1 & 0.8 & 0 & 0 & 0.816 \\
\hline SSEs, n (\%) & 9 & 7.1 & 2 & 3.7 & 0.383 \\
\hline SSOs & 8 & 6.3 & 2 & 3.7 & 0.484 \\
\hline Seroma & 7 & 5.5 & 1 & 1.9 & \\
\hline Hematoma & 2 & 1.6 & 1 & 1.9 & \\
\hline SSIs & 2 & 1.6 & 0 & 0 & 0.354 \\
\hline Cellulitis & 1 & 0.8 & 0 & 0 & \\
\hline Superficial & 1 & 0.8 & 0 & 0 & \\
\hline SSOPI & 1 & 0.8 & 0 & 0 & 0.513 \\
\hline
\end{tabular}

rTAPP. robotic transabdominal preperitoneal; rTEP-RS: robotic total extraperitoneal Rives-Stoppa; CCI® Comprehensive Complication Index (University of Zurich, Zurich, Switzerland); SSEs: surgical site events; SSOs: surgical site occurrences; SSIs: surgical site infections; SSOPI: surgical site occurrence or infection requiring procedural intervention; SD: standard deviation; IQR interquartile range. 
Table 4. Logistic regression analysis results

\begin{tabular}{llccc} 
Variable & $\mathbf{p}$ & Odds ratio & \multicolumn{2}{c}{ 95\% confidence interval } \\
\cline { 4 - 5 } & & & Lower bound & Upper bound \\
\hline Age (year) & 0.223 & 1.023 & 0.987 & 1.060 \\
Sex (female) & $\mathbf{0 . 0 4 9}$ & 2.820 & 1.003 & 7.965 \\
Console time (minute) & $\mathbf{0 . 0 1 5}$ & 1.018 & 1.004 & 1.033 \\
Incarceration (small bowel) & 0.117 & 3.810 & 0.716 & 20.260 \\
\hline
\end{tabular}

\section{Discussion}

According to the International Endohernia Society (IEHS) guidelines, ${ }^{[12]}$ laparoscopic TAPP and TEP techniques are feasible options for the repair of small- and medium-sized primary and incisional abdominal wall hernias, with minimal morbidity. This is attributed to minimal trauma, standard mesh use with reduced fixation, limited entry into the abdominal cavity, the ability to excise the hernia sac and close defects with anatomical reconstruction of the abdominal wall. However, these techniques have been criticized for the need of extensive surgical experience to perform safely, especially when dissecting the preperitoneal and retromuscular planes. These challenges may be more prominent with laparoscopic repair, as mobility is limited and surgical steps such as intracorporeal defect closure are more difficult. ${ }^{[13]}$ The dexterity and facilitated dissection afforded by the robotic platform may help mitigate these challenges, as demonstrated by recent studies. ${ }^{[4,14]}$ To our knowledge, this is the largest study comparing rTAPP and rTEP-RS techniques.

The main advantage of preperitoneal and retrorectus mesh placements is that they allow for a natural anatomical barrier between the mesh and abdominal viscera, thus avoiding potential future complications. This also allows for the use of non-coated mesh which is more cost-effective. Each of these mesh placements has its own benefits as well. With TEP-RS repair, placing the mesh in the vascularized retromuscular plane aids in clearance of infection and avoids the need for mesh fixation since the mesh is held firmly in place by the rectus muscle and its fascia. ${ }^{[15]}$ Moreover, the TEP-RS technique drastically decreases the need for adhesiolysis which is associated with intraoperative bowel injury, seroma formation, and postoperative morbidity. ${ }^{[16-18]}$ Furthermore, surgeons can take advantage of a large plane which can accommodate larger mesh sizes. Ultimately, this leads to a larger mesh-to-defect ratio, which is a crucial determinant of hernia recurrence.
[19] Criticisms of the TEP-RS approach, however, include longer operative times and the need for precise dissection while developing the retromuscular plane. This is important since there is a risk of intraoperative bowel injury, especially during crossover into the contralateral rectus sheath and in patients with incisional hernias. ${ }^{[4]}$ On the other hand, the TAPP approach is more familiar for surgeons with previous IPOM experience, and although involves intraabdominal adhesiolysis, is less time-consuming than the TEP-RS approach. Both these procedures can be achieved robotically, and the safety and feasibility of these robotic techniques have been previously demonstrated. In a study with 52 patients undergoing rTEP repair, including 20 patients with adjunctive TAR, the authors encountered no intraoperative complications or conversions, 3 self-resolving SSOs, and a mean hospital LOS of 0.71 days. ${ }^{[20]}$ Similarly, the authors conducted another study involving 54 rTAPP hernia repairs with various anatomical locations and etiologies, and found no intraoperative complications, 2 clinically significant SSOs, and that nearly all patients were discharged on the same day of operation. ${ }^{[14]}$

Belyansky et al. ${ }^{[4]}$ described their robotic TEP technique with selective TAR utilization among 37 patients. From the patients undergoing rTEP-RS repair $(n=29)$, the average hernia defect size (greatest dimension) was found to be $5.9 \mathrm{~cm}$ with a corresponding average mesh area of $532 \mathrm{~cm}^{2}$. The average operative time was 141.3 minutes. In Kennedy et al.'s ${ }^{[5]}$ study comparing rTAPP $(n=36)$ and rIPOM $(n=27)$ procedures, they reported an average defect size (largest diameter) of $3.98 \mathrm{~cm}$ and a mean operative time of approximately 159 minutes for the rTAPP group. In this study, we found several notable differences between our two groups' intraoperative variables. Average defect area was significantly larger in the rTEPRS group as compared to the rTAPP group (15.7 vs 3.1 $\left.\mathrm{cm}^{2}, \mathrm{p}<0.001\right)$. Consequently, average mesh size was 
also larger in the rTEP-RS group (225 vs $113 \mathrm{~cm}^{2}$ ) while mesh-to-defect ratios favored the rTAPP group (19.2 vs 11.5) $(\mathrm{p}<0.001)$. While the rTEP-RS technique allows for a wide retromuscular dissection to accommodate larger mesh sizes, it is more challenging to develop as large of a space in the preperitoneal plane. This is mainly due to the tight adherence of abdominal wall layers near the linea semilunaris. This partially explains why the rTAPP technique was chosen for smaller hernias, in order to maintain adequate mesh-to-defect ratio and mesh overlap. $76.2 \%$ of patients in the rTAPP group underwent mesh fixation as compared to $4.8 \%$ in the rTEP-RS group $(\mathrm{p}<0.001)$. Despite this, mean skin-to-skin times were longer in the rTEP-RS group (72 vs 56 minutes, $\mathrm{p}<0.001$ ), likely due to the more time-consuming dissection secondary to larger defects in the rTEP-RS group. Primary defect closure was achieved in $93 \%$ and $100 \%$ of the rTAPP and rTEP-RS groups, respectively. As mentioned previously, defect closure is challenging with laparoscopic approaches, even when incorporating TAR. In this study, the high rate of defect closure is likely owed to the robotic technology.

Comparisons between TAPP and TEP approaches have been discussed for inguinal hernia repairs (IHR). Wei et al. ${ }^{[21]}$ conducted a meta-analysis comparing laparoscopic TAPP and TEP techniques for IHR from 10 randomized controlled trials. A total of 1047 patients were included and they found no difference between the two procedures in terms of hernia recurrence, pain scores, operation time, hospital stay, or total complications. They recommended that due to the increased complexity of TEP, inexperienced surgeons should attempt TAPP first, while taking into account patient characteristics regardless of technique choice. Of note, although pain scores did not differ between our study groups, a higher average MME was observed in the rTAPP group. Kennedy et al. ${ }^{[5]}$ reported 30-day outcomes in their study with one postoperative hematoma in the rTAPP group and one readmission unrelated to the procedure. In Belyansky et al.'s study, ${ }^{[4]}$ the mean LOS for the RS subgroup was 0.3 days and within a 30-day follow-up period, no patients experienced wound-related complications. The mean LOS for this study's rTAPP group was 0.09 days as compared to 0.27 days for the rTEP-RS group. Throughout our study's 90-day follow-up period, the rTAPP group had a higher overall complication rate (11\% vs $7.4 \%)$, including a higher rate of major complications (3 vs 0), SSEs (9 vs 2), and SSOPIs (1 vs 0 ). However, none of these differences were statistically significant.
Our multivariate regression yields some interesting findings. In terms of our patient characteristics, age was found to be different between the two groups in a univariate analysis. However, only female gender was identified in the regression to be associated with postoperative complications. This may be due to the fact that the proportion of incisional hernias was higher among female patients ( $\mathrm{p}=0.049 ; 31.8 \%$ vs 18.6 ), although hernia etiology as a separate variable did not fit into the regression model. Although small bowel incarceration is generally associated with postoperative complications, this was not the case in our study ( $p=0.177)$. On the other hand, there was a significant difference in console time between the two study groups, with clinically negligible association with postoperative complications $(\mathrm{OR}=1.018)$.

There are some limitations in this study. Although our data was recorded prospectively, the study's retrospective structure can be considered a limitation. In order to reduce potential to reduce the effect of potential bias, we performed a regression analysis. Moreover, a greater number of patients in the rTEP-RS group could help observe more differences between the two techniques. Another limitation is that this was a single-center study which limits its generalizability. Multicenter studies that represent more diverse surgeon experience are needed. Other study limitations include the absence of patient-reported outcomes, such as pain assessment and quality of life, and the lack of long-term follow-up outcomes to evaluate repair durability.

In conclusion, the short-term results for rTEP-RS repair were similar rTAPP repair. The rTEP-RS approach allowed for large hernias defects to be repaired with large-sized mesh. Moreover, rTEP-RS is associated with longer operating times likely due to larger defects.

\section{Disclosures}

Preliminary results of this study was accepted as an oral presentation by 42nd Annual European Hernia Society Congress committee.

Ethichs Committee Approval: The study was approved by the Local Ethics Committee (IRB\#:HW202, Good Samaritan Medical Center).

Peer-review: Externally peer-reviewed.

Conflict of Interest: None declared. 
Authorship Contributions: Concept - O.Y.K., F.G.; Design - N.B.A., F.G., O.Y.K.; Supervision - O.Y.K., F.G.; Materials - O.Y.K., F.G., N.B.A.; Data collection and/or processing F.G., O.Y.K., N.B.A.; Analysis and/ or interpretation - F.G., N.B.A., O.Y.K.; Literature search - N.B.A., F.G.; Writing N.B.A., F.G., O.Y.K.; Critical review - O.Y.K., N.B.A., F.G.

\section{References}

1. LeBlanc KA, Booth WV. Laparoscopic repair of incisional abdominal hernias using expanded polytetrafluoroethylene: preliminary findings. Surg Laparosc Endosc 1993;3:39-41.

2. Muysoms FE, Bontinck J, Pletinckx P. Complications of mesh devices for intraperitoneal umbilical hernia repair: a word of caution. Hernia 2011;15:463-8. [CrossRef]

3. Chelala E, Baraké H, Estievenart J, Dessily M, Charara F, Allé JL. Long-term outcomes of 1326 laparoscopic incisional and ventral hernia repair with the routine suturing concept: a single institution experience. Hernia 2016;20:101-10. [CrossRef]

4. Belyansky I, Reza Zahiri H, Sanford Z, Weltz AS, Park A. Early operative outcomes of endoscopic (eTEP access) roboticassisted retromuscular abdominal wall hernia repair. Hernia 2018;22:837-47. [CrossRef]

5. Kennedy M, Barrera K, Akelik A, Constable Y, Smith M, Chung $P$, et al. Robotic TAPP Ventral Hernia Repair: Early Lessons Learned at an Inner City Safety Net Hospital. JSLS 2018;22:e2017.00070. [CrossRef]

6. Kudsi OY, Gokcal F, Chang K. Robotic intraperitoneal onlay versus totally extraperitoneal (TEP) retromuscular mesh ventral hernia repair: A propensity score matching analysis of shortterm outcomes. Am J Surg 2020;220:837-44. [CrossRef]

7. Gokcal F, Morrison S, Kudsi OY. Short-term comparison between preperitoneal and intraperitoneal onlay mesh placement in robotic ventral hernia repair. Hernia 2019;23:957-67.

8. Muysoms FE, Miserez M, Berrevoet F, Campanelli G, Champault GG, Chelala $E$, et al. Classification of primary and incisional abdominal wall hernias. Hernia 2009;13:407-14.

9. Dindo D, Demartines N, Clavien PA. Classification of surgical complications: a new proposal with evaluation in a cohort of 6336 patients and results of a survey. Ann Surg 2004;240:205-13. [CrossRef]
10. Slankamenac K, Graf R, Barkun J, Puhan MA, Clavien PA. The comprehensive complication index: a novel continuous scale to measure surgical morbidity. Ann Surg 2013;258:1-7. [CrossRef]

11. Petro CC, Novitsky YW. Classification of hernias. In: Novitsky YW, editor. Hernia Surgery. Switzerland: Springer; 2016. p. 15-21. [CrossRef]

12. Bittner R, Bingener-Casey J, Dietz U, Fabian M, Ferzli G, Fortelny $\mathrm{R}$, et al. Guidelines for laparoscopic treatment of ventral and incisional abdominal wall hernias (International Endohernia Society [IEHS])-Part III. Surg Endosc 2014;28:380-404.

13. Belyansky I, Daes J, Radu VG, Balasubramanian R, Reza Zahiri $\mathrm{H}$, Weltz AS, et al. A novel approach using the enhanced-view totally extraperitoneal (eTEP) technique for laparoscopic retromuscular hernia repair. Surg Endosc 2018;32:1525-32.

14. Orthopoulos G, Kudsi OY. Feasibility of Robotic-Assisted Transabdominal Preperitoneal Ventral Hernia Repair. J Laparoendosc Adv Surg Tech A 2018;28:434-8. [CrossRef]

15. Novitsky YW, Fayezizadeh M, Majumder A, Neupane R, Elliott $\mathrm{HL}$, Orenstein SB. Outcomes of Posterior Component Separation With Transversus Abdominis Muscle Release and Synthetic Mesh Sublay Reinforcement. Ann Surg 2016;264:22632. [CrossRef]

16. Huang CC, Lien HH, Huang CS. Long-term follow-up of laparoscopic incisional and ventral hernia repairs. J Laparoendosc Adv Surg Tech A 2013;23:199-203. [CrossRef]

17. Mavros MN, Velmahos GC, Larentzakis A, Yeh DD, Fagenholz $\mathrm{P}$, de Moya $\mathrm{M}$, et al. Opening Pandora's box: understanding the nature, patterns, and 30-day outcomes of intraoperative adverse events. Am J Surg 2014;208:626-31. [CrossRef]

18. Mavros MN, Velmahos GC, Lee J, Larentzakis A, Kaafarani HM. Morbidity related to concomitant adhesions in abdominal surgery. J Surg Res 2014;192:286-92. [CrossRef]

19. Tulloh B, de Beaux A. Defects and donuts: the importance of the mesh:defect area ratio. Hernia 2016;20:893-5. [CrossRef]

20. Kudsi OY, Gokcal F. Lateral approach totally extraperitoneal (TEP) robotic retromuscular ventral hernia repair. Hernia 2019 Nov 27 [Epub ahead of print], doi: 10.1007/s10029019-02082-9. [CrossRef]

21. Wei FX, Zhang YC, Han W, Zhang YL, Shao Y, Ni R. Transabdominal Preperitoneal (TAPP) Versus Totally Extraperitoneal (TEP) for Laparoscopic Hernia Repair. A Meta-Analysis. Surg Laparosc Endosc Percutan Tech. 2015;25(5):375-83. [CrossRef] 